\title{
Effects of Irregular Shifts on Incidence of Type II Diabetes Among Healthcare Workers
}

\author{
Varun Rameswara ${ }^{1}$, Amy Blevins ${ }^{2}$, George Eckert ${ }^{3}$, Denis Jusufbegovic ${ }^{4}$ and Ashay \\ Bhatwadekar $^{4}$ \\ ${ }^{1}$ Indiana University School of Medicine; ${ }^{2}$ Ruth Lilly Medical Library; ${ }^{3}$ Department of Biostatistics; \\ ${ }^{4}$ Department of Ophthalmology, Indiana University School of Medicine
}

\section{Background/Objective:}

The prevalence of shift work ranges around $20 \%$ among American and European workers and is significantly higher in the healthcare field. Variable work schedules among shift workers are associated with adverse health effects, including the increased risk of metabolic disorders and obesity. This systematic review aims to evaluate the association between shift work and type 2 diabetes/insulin resistance.

\section{Methods:}

This research is part of a more extensive systematic review that has been registered on Prospero. Due to time constraints, we began with a scoping search with the goal of completing a rapid review. Searches were completed using the Ovid and PubMed databases with the following keywords and subject headings: Health Personnel; Shift Work Schedule; Circadian Rhythm; Work Schedule Tolerance; Metabolic Diseases; Overweight; Glucose Metabolism Disorders; Lipid Metabolism Disorders; Malabsorption Syndromes; Metabolic Syndrome; and Diabetes Mellitus. The resulting articles were uploaded onto Covidence for screening and data extraction. After screening the abstracts for diabetes and insulin resistance, eight articles were selected for the full-text screening of which data extraction was performed on three.

\section{Results:}

Overall, 163,555 participants performing evening, night, rotating shifts, or regular shifts were identified in the three studies. The average age of study participants was found to be $44.0 \mathrm{yrs}$, 163,422 were females (>99.9\%), and of the total participants, 98,303 subjects were shift workers $(60.10 \%)$. For healthcare shift workers, there was a significant association between shift work and diabetes across all three studies; however, the effect was modest (relative risk in the range of 1.19-1.26).

\section{Conclusion:}

There is a positive relationship between shift work and diabetes among healthcare workers. Therefore, strategies focusing on reducing shift work or treating these workers as a high-risk group for diabetes by taking measures to minimize other exposures will be beneficial in reducing the incidence of diabetes among this population. 